УДК 621.18

\title{
ПОДХОДЫ К ЧИСЛЕННОМУ ИССЛЕДОВАНИЮ ТОПОЧНЫХ КАМЕР С ЦИРКУЛИРУЮЩИМ КИПЯЩИМ СЛОЕМ
}

\author{
Гиль Андрей Владимирович1, \\ angil@tpu.ru
}

\section{Заворин Александр Сергеевич 1 , kotel@tpu.ru}

\author{
Кокшарев Олег Максимович1, \\ omk2@tpu.ru
}
1 Национальный исследовательский Томский политехнический университет, Россия, 634050, г. Томск, пр. Ленина, 30.

\begin{abstract}
Актуальность исследования. В современных научных исследованиях вычислительный эксперимент является одним из наиболее продуктивных средств изучения комплекса задач, в которых результат зависит от одновременного проявления аэродинамики, теплообмена и горения. Численные расчеты при этом позволяют не только правильно интерпретировать фризические явления, фриксируемые на экспериментальных установках, но нередко и дополнить существенно более дорогостоящий и трудоемкий физический или натурный эксперимент компьютерной симуляцией. В связи с этим актуальность проведения исследований, направленных на совершенствование методов численного анализа топочных процессов в котлах с циркулирующим кипящим слоем на основе компьютерного математического моделирования, является весьма высокой для обоснования основных проектно-конструкторских и технологических решений, принимаемых при создании котлов с циркулирующим кипящим слоем. Помимо практической значимости, такого рода исследование обладает высокой научной составляющей, поскольку в основе поставленной задачи лежат фундаментальные закономерности физико-химических процессов в циркулирующем кипящем слое.

Цель: определение подходов и применимости численных алгоритмов с удовлетворительным уровнем детализации и сходимости для моделирования топочных процессов в котлах с циркулирующим кипящим слоем.

объекты: топка с циркулирующим кипящим слоем, оборудованная двумя вводами подачи топлива и инертных частии на тыльной стене и десятью трубопроводами вторичного дутья на фронтовой стене в два яруса.

Методы: математическое моделирование физико-химических процессов в топочной камере с циркулирующим кипящим слоем на основе Эйлеро-Эйлерова и Эйлеро-Лагранжева подходов с использованием RANS моделей. Записаны диффференциальные уравнения для сохранения массы, импульса, энергии и переноса частиц. Численное исследование проведено с использованием пакета прикладных программ Ansys Fluent v.12.1
\end{abstract}

Результаты. Определены начальные и граничные условия для численного моделирования процессов в топочной камере котельного агрегата с циркулирующим кипящим слоем при использовании Эйлеро-Эйлерова и Элеро-Лагранжева подходов.

\section{Ключевые слова:}

Котел с циркулирующим кипящим слоем, математическое моделирование,

метод Эйлера-Эйлера, метод Эйлера-Лагранжа, физико-химические процессы.

\section{Введение}

Начиная с последней трети прошлого века развитие новых технологий сжигания твердого топлива оказалось сопряжено с тенденцией повышения экологических параметров и обеспечения маневренности топливосжигающих агрегатов при постоянном ухудшении качества твердых топлив.

Именно поэтому в 1970-1980 гг. усилиями нескольких инжиниринговых компаний получила практическое применение перспективная технология циркулирующего кипящего слоя (ЦКС), которая и по настоящее время играет важную роль в развитии технологий для генерации энергии с минимальными выбросами вредных веществ.

Данный способ представляется перспективным, поскольку успешно позволяет решать задачи повышения эффективности выгорания низкокачественного, высокозольного и влажного топлива [1], снизить выбросы золовых частиц, окислов азота и серы, а также повысить возможность последующего использования золошлаковых отходов.
На современном этапе мировой опыт эксплуатации котлов с ЦКС очень богат и постоянно совершенствуется. За годы использования ЦКС в энергетике удалось серьезно уменьшить металлоемкость, усовершенствовать различные конструктивные решения, повысить уровень надежности и экономичности.

Однако в данный момент как в России, так и в зарубежных странах возможности численного прогнозирования процессов в слое и образования тех или иных веществ в результате сжигания в ЦКС все еще далеки от завершения. Например, в России представлен только единственный образец котла с ЦКС (Новочеркасская ГРЭС-9), а газодинамические процессы в топочных камерах с ЦКС остаются недостаточно глубоко изученными, что отчасти обусловлено и отсутствием единой нормативной методики инженерных расчетов. В совокупности названное отставание сдерживает развитие отечественного котлостроения и теплоэнергетики в целом. Поэтому одно из направлений Энергетической стратегии России до 2030 г. [2] сконцентрировано на создании отечественных котлов 
с ЦКС, которые обладают преимуществами перед наиболее распространенным факельным сжиганием в пылеугольных котлах, конструкции которых спроектированы в то время, когда не было «жестких» экологических ограничений.

Из-за нехватки опыта эксплуатации и проектирования котлов с ЦКС проведение натурных экспериментов затруднено, а выполнение исследований на лабораторных установках позволяет изучить и оценить влияние лишь одного или нескольких параметров, а не совокупность процессов в целом.

Поэтому на современном этапе исследований в технических науках вычислительный эксперимент является одним из наиболее продуктивных средств изучения комплекса задач, в которых результат зависит от одновременного проявления аэродинамики, теплообмена и горения. Численные расчеты при этом позволяют не только правильно интерпретировать физические явления, фиксируемые на экспериментальных установках, но нередко и дополнить существенно более дорогостоящий и трудоемкий физический или натурный эксперимент компьютерной симуляцией. Соответственно актуальность проведения исследований, направленных на совершенствование методов численного анализа топочных процессов в котлах с ЦКС на основе компьютерного математического моделирования, является весьма высокой.

\section{Проблематика. Практика и методология исследования феномена}

Типичная схема котла ЦКС представлена на рис. 1 [3]. Слой из твердого материала ожижается первичным воздухом, который поступает в топку через нижнюю решетку. Псевдоожиженный слой, как правило, представляет собой смесь золы, недогоревшего углеродистого остатка, инертного вещества (обычно песок) и возможно добавление сорбента. Подача топлива и других твердых компонентов осуществляется в нижней части топки. Вторичный воздух вводится над точками подачи топлива в различных местах и на различных высотах для выполнения поэтапного сжигания. Температура в топке ниже температуры агломерации твердых веществ, как правило, в диапазоне $750-950{ }^{\circ} \mathrm{C}$, т. е. значительно ниже, чем при сжигании пылеугольного топлива факельным способом или при сжигании на колосниковой решетке.

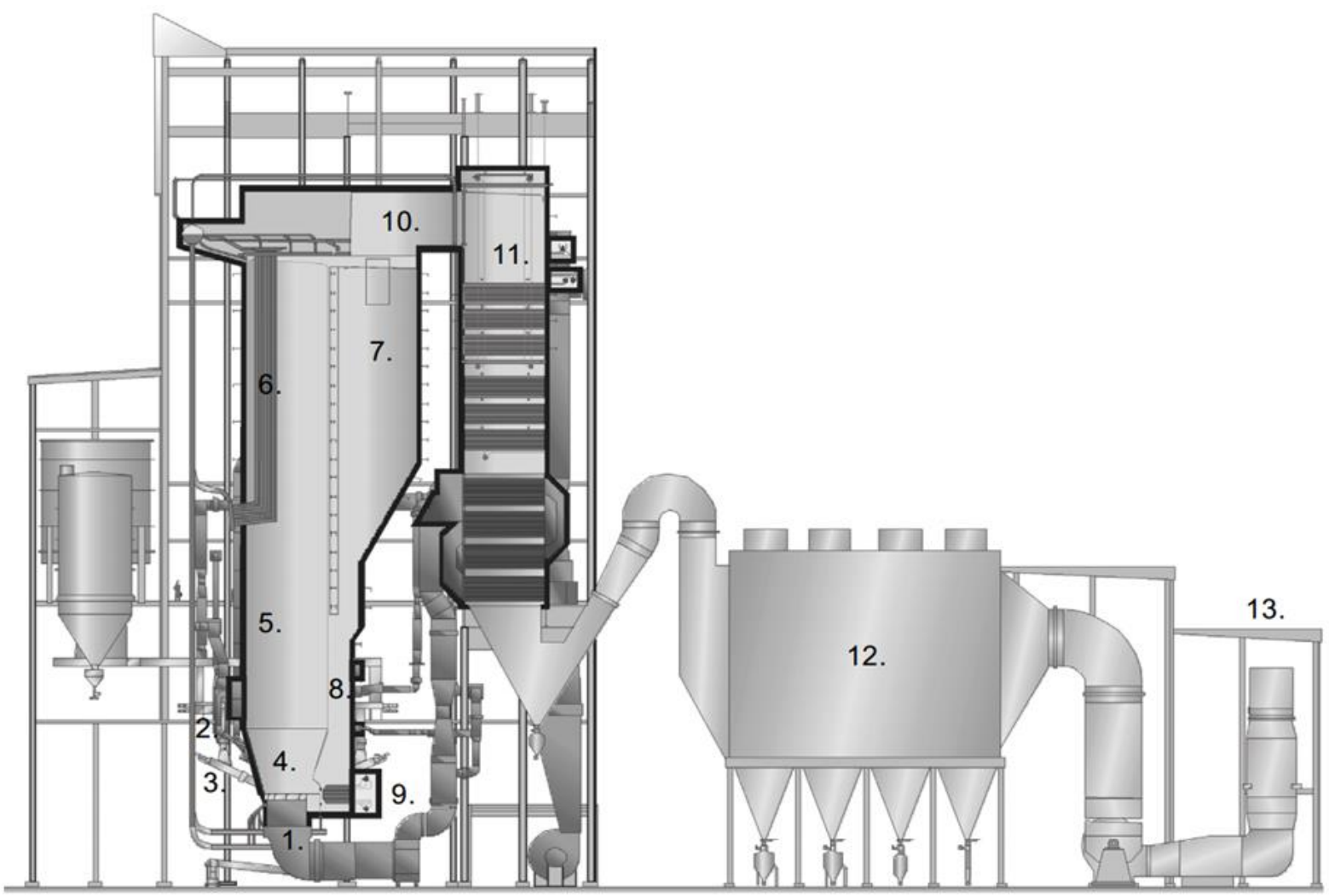

Рис. 1. Типичная компоновка котла с циикулирующим кипящим слоем (компания Foster Wheeler Energia Oy): 1 - nервичный воздух, 2 - вторичный воздух, 3 - топливо, известняк, добавочное питание, 4 - нижняя часть топки с огнеупорной футеровкой, 5 - мембранные экраны топки, 6-внутренние поверхности нагрева, 7 - сепаратор (циклон), 8 -система возврата частии, 9 - внешний кипящий теплообменник, 10 - горизонтальный газоход, 11 - опусконой газоход с теплообменниками, 12 - электрофильтр, 13 - дымовая труба

Fig. 1. Typical arrangement of circulating fluidized bed boiler (Foster Wheeler Energia Oy): 1 - primary air, 2 - secondary air, 3 - fuel, limestone, additional feed, 4 - lower part of the furnace with refractory lining, 5 - membrane screens of the furnace, 6 - internal heating surfaces, 7 - separator (cyclone), 8 -particle return system, 9 - external fluidized heat exchanger, 10 - horizontal flue, 11 -down flue with heat exchangers, 12 - electrofilter, 13 - chimney 
Обозначенные выше исследования непосредственно связаны с численным моделированием статических и динамических процессов на модульноструктурированной основе, которые отражают эффективность работы проектируемого котельного агрегата. Такие модели должны основываться на детальных механизмах реакций и помимо гомогенных и гетерогенных реакций включать в себя также и каталитические реакции, затрагивая многообразие других явлений, в реальности протекающих и описывающих котельную установку. В настоящее время многие ученые уже подтвердили тот факт, что развитие микроскопических подходов в CFD моделировании (Computational Fluid Dynamics - вычислительная гидрогазодинамика) страдает от нехватки и трудности получения соответствующих экспериментальных данных от объектов промышленной эксплуатации ЦКС, а также от длительного времени расчета. Поэтому одно из направлений, которое сложилось из-за больших габаритных размеров установок ЦКС в совокупности со сложной системой уравнений, требует моделей, которые будут позволять разрешать данное явление как можно более ресурсоэффективней и с достаточной точностью на грубой расчетной сетке с применением эмпирических или полуэмпирических выражений для анализа процесса, увеличенным временным шагом или в стационарных условиях (макроскопическое моделирование). Как видно, сочетание моделей CFD с полным описанием химических процессов является слишком сложной задачей с точки зрения вычислений, и поэтому ведется разработка упрощенных подходов. По мнению различных исследователей, в ближайшем будущем процессы в ЦКС в промышленном масштабе не представляется возможным смоделировать с полным набором уравнений, описывающих химические процессы, тепломассоперенос и гранулометрический состав частиц [4-9]. Тем не менее учеными $[10,11]$ проводятся исследования влияния конструкций циклонных установок на гидродинамику потока в котле с ЦКС в трехмерной (3D) постановке задачи с полным циклом протекания внутренних процессов. Из анализа расположения циклонов было установлено, что распределение потока частиц протекает более равномерно при осесимметричном расположении, чем при расположении относительно центральной оси. Исходя из этого, моделирование полного цикла является перспективным способом для обеспечения качественных расчетов на этапе проектирования.

В настоящее время используются два основных подхода для моделирования газовых потоков с наличием твердых частиц, а именно Эйлеров-Лагранжев (ЭЛ) и Эйлеров-Эйлеров (ЭЭ) подход. ЭЛ подход, который рассматривает частицы индивидуально, часто применяется для описания систем методом траекторий [12]. С помощью этого метода вычисляются траектории отдельных частиц или частиц, представляющих большое число отдельных субчастиц, пренебрегая их столкновениями. Для более высоких концентраций твердых частиц, в которых существуют столкновения между частицами, доминируют перенос и дисперсия импульса. Для этих явлений может быть использован метод дискретного моделирования частиц (DPM - Discrete Phase Model) [13]. При использовании метода DPM вычисляется траектория каждой отдельной частицы, предсказываются столкновения между отдельными частицами, и учитывается их влияние на траектории друг друга. Этот метод требует много вычислительных ресурсов и потому ограничен на текущий момент числом частиц $10^{6}$. По этой причине DPM пока нельзя использовать для моделирования крупномасштабных систем ЦКС, которые насчитывают в себе более чем $10^{12}$ частиц.

Модель промышленного образца котла с ЦКС была смоделирована в [14] с помощью модели DDPM (DDPM - Dense Discrete Phase Model) для изучения процесса горения угля и гидродинамики частиц. Исследования процесса горения угля в ЦКС при различных концентрациях О2 с использованием модели DDPM, а также влияние гранулометрического состава частиц и концентрации $\mathrm{O} 2$ на структуру дисперсного потока и характеристики горения были рассмотрены в [15].

Наиболее широко используется ЭЭ подход для моделирования котлов с ЦКС. В методе ЭЭ (двухжидкостной модели) газовая и твердая фаза рассматриваются как два полностью взаимопроникающих континуума. Используемые уравнения являются обобщением уравнений Навье-Стокса для взаимодействующих сред [16]. Частицы внутри твердой фазы рассматриваются с идентичными и определенными диаметрами и плотностью. Для моделирования распределения частиц по размерам необходимо использовать несколько псевдожидкостей для моделирования твердой фазы, каждая из которых представляет один класс частиц $[17,18]$.

В работах [19] и [20] процесс смешения описывается моделью $1,5 \mathrm{D}$. Однако детально рассматривают химические процессы окисления метана, этана, $\mathrm{HCN}$ и NH3 так же, как и взаимодействия углеводородного сырья (CHi, HCCO), компонентов азота (NO, NHi, N2) и $\mathrm{H} 2$.

Среди различных попыток сформировать поток частиц кинетическая теория гранулированных потоков (KTGF - Kinetic Theory of Granular Flow) нашла свое широчайшее применение. Она является дополнением к классической кинетической теории газов, описанной в [21], и применяется к плотным потокам частиц, описывая их столкновения [16]. Случайное распределение и упругие столкновения твердых частиц делают кинетическую теорию идеально подходящей для описания зернистых течений.

Столкновения частиц и воздействия на твердые фазы не единственные проблемы моделей «газтвердая частица». Другой проблемой моделирования является использование соответствующих корреляций сопротивления для описания обмена импульсами между двумя фазами. В [22] выявили, что воздействие на твердые частицы, предсказанное с помощью модели KGTF, не оказывает существенного влияния в общую структуру потока, в то время как влияние кла- 
стеризации частиц и, следовательно, трение имеет доминирующую роль в результатах моделирования.

Межфазный перенос импульса является одним из наиболее значимых членов в уравнениях импульса газа и твердой фазы. Этот обмен импульсом представлен силой сопротивления. Сила сопротивления на одной сферической частице хорошо коррелирует для широкого диапазона чисел Рейнольдса [23]. Однако эти корреляции теряют свою актуальность в твердогазовой суспензии, поскольку на силу сопротивления одной частицы влияет наличие других частиц [24].

Для охвата широкого диапазона концентраций твердых веществ при моделировании CFD довольно часто используется сочетание корреляций С. Эргуна [25] и Вена и Ю [26]. Эти корреляции были первоначально разработаны на основе экспериментов с гомогенными системами. Однако распределение в псевдоожиженных слоях гетерогенно.

CFD моделирование с этими или подобными корреляциями сопротивления, как правило, хорошо согласуется с экспериментальными результатами для верхних разреженных областей подъемного газохода ЦКС. Однако чаще всего моделирование не позволяет описать нижнюю часть подъемного газохода, где в большинстве экспериментов наблюдается область с высокими концентрациями твердых частиц. Обычно при моделировании специально занижают концентрацию твердых веществ в данной области. Например, в [27] описывается, что корреляции сопротивления, полученные из уравнения Эргуна, вызвали быстрый перенос твердых тел и в результате моделируемый поток стал довольно разбавленным в целом. Причиной такого поведения является монотонное увеличение сопротивления с возрастанием концентрации твердых тел, что контрастирует с экспериментально наблюдаемым уменьшением сопротивления за счет образования кластеров.

Моделирование топочных камер с ЦКС по методу решеточных уравнений Больцмана (Lattice Boltzmann Method), в работе [28] показано, что расположение частиц сильно влияет на сопротивление суспензий, поэтому при расчете обмена импульсами необходимо учитывать структуру суспензий. Метод имеет преимущества над другими методами (например, finite element method) по возможности моделирования многофазных потоков и моделирования в пористых средах, а также по меньшей трудоемкости распараллеливания. Немаловажно и то, что вычислительный алгоритм состоит только из простейших арифметических операций. Данный метод - один из новых, поскольку основанные на нем первые коммерческие продукты начали появляться около 2010 г.

Некоторые недавние исследования учитывают локальную неоднородность потока «газ-твердая частица» в ЦКС для расчета силы сопротивления. Первые подходы к учету структуры потока на подсеточном масштабе были сделаны в [29] и [30]. Последние два коллектива вычисляли сопротивление на основе теории мультимасштабного минимума потенциальной энергии (EMMS - Energy-Minimization Multi-Scale) [31].
В статье [32] уделяется внимание модели стационарного процесса в котле с ЦКС на основе гидродинамики, теплообмена и горения. Эта модель прогнозирует температуру дымовых газов, концентрацию компонентов $\left(\mathrm{O}_{2}, \mathrm{H}_{2} \mathrm{O}, \mathrm{CO}, \mathrm{CO}_{2}\right.$ и $\left.\mathrm{SO}_{2}\right)$ и распределения концентрации продуктов сгорания как в осевых, так и радиальных местах вдоль топки, включая нижнюю и верхнюю части. Модель была верифицирована на основе экспериментальных данных, полученных в котле мощностью 35 т/ч с низким коэффициентом циркуляции.

А. Гунгор [33] представил свои исследования по разработке динамической двумерной модели с учетом гидродинамического поведения ЦКС. Моделирование производится на подъемной трубе, имитирующей ЦКС, с анализом в двух зонах: нижняя зона в режиме турбулентной флюидизации моделируется как двухфазный поток, который подразделяется на пузырьковую твердую фазу (без включения твердых частиц) и твердую эмульсионную фазу (с включениями твердых частиц). Моделирование учитывает осевое и радиальное распределение пустотности, скорости и перепада давления для газа и твердой фазы, а также объем твердой фракции и распределения частиц для твердой фазы. Результаты моделирования сравниваются и подтверждаются на основании экспериментальных данных, приведенных в литературе.

Х. Накамура в своем исследовании [34] представил результаты моделирования поведения частицы во вращающемся псевдоожиженном слое. Предложенная численная модель была основана на модели, объединяющей DEM (метод дискретных элементов) и CFD. Движение жидкости вычислялось двумя измерениями путем решения локальных усредненных базовых уравнений. В предложенной модели были одновременно проведены расчеты движения жидкости по методу CFD и движения частиц по методу DEM.

А. Хадаванд [35] описал математическую модель сжигания в ЦКС на основе уравнений сохранения массы и энергии. Используя эти зависимости, на основе подпространственного метода получена динамическая модель фазового пространства, ориентированная на температуру слоя. Температура слоя, которая влияет на общую эффективность котла и уровень выбросов загрязняющих веществ, является одним из наиболее значимых параметров в работе этого типа систем. Из-за наличия динамической и параметрической неопределенности в модели был применен надежный алгоритм управления на основе линейных матричных неравенств (ЛМН) для того, чтобы контролировать температуру слоя с использованием исходных данных, например величины расхода угля и скорости псевдоожижения.

Л. Конг и П. Ходжсон [36] работали над улучшением понимания и поиском надежной и простой модели теплопередачи. Поток газа и теплоперенос между псевдоожиженными слоями и поверхностями погруженного объекта численно моделируются на основе парной модели «частица-слой» и «пористая среда». Парная модель позволяет моделировать течения газа и теплообмен вблизи поверхности погруженного объ- 
екта в псевдоожиженном слое и успешно применяется при расчете динамических характеристик газовой фазы, изменения температуры частиц и радиационных параметров групп частиц.

Ж. Бастос в своем исследовании [37] изучал радиальные профили скорости частиц, которые были разбиты на семь осевых уровней в подъемной трубе высоконапорного циркулирующего кипящего слоя (HFCFB), используя двухфазную 3D модель вычислительной гидродинамики (CFD).

Д. Корнелиссен [38] в своем исследовании применял многожидкостную модель Эйлера в модели CFD с расширением гранулированного потока для моделирования жидко-твердого циркулирующего кипящего слоя. Численное моделирование оценивается качественно по экспериментальным данным из литературы и количественно по сравнению с новыми экспериментальными данными. Исследован эффект размера сетки, временного шага и критериев сходимости.

На примере котла с ЦКС, установленного на ТЭЦ Лагижа в Польше, предлагаются два подхода решения задачи моделирования - ЭЭ и ЭЛ, с расширенным методом мультифазной частицы в ячейке (MPPIC), а также их сравнение [39]. Модели, основанные на кинетическом представлении потока частиц, описывают взаимодействие между частицами [40]. Лучистый теплообмен моделировался с помощью метода дискретных ординат. Излучающие свойства $\mathrm{CO} 2$ и $\mathrm{H} 2 \mathrm{O}$ были рассчитаны с использованием модели взвешенной суммы серых газов (WSGGM - weighted sum of gray gases model) с использованием различных модельных подходов, в том числе модели, предложенной М. Бордбаром в [41].

Метод мультифазной частицы в ячейке, или гибридный метод Эйлера-Лагранжа, использует М. Вэнг в [42] для моделирования котла с ЦКС мощностью 250 МВт. Данная модель предполагает моментальное испарение влаги и выход летучих топлива В состав реакций данной модели входят газификация и горение угля (углерода), реакция конверсии окиси углерода в двуокись углерода, сжигание нелетучих гидрокарбонатов и горение окиси углерода. Также представлены профили температуры, а применяемые модели теплопередачи не описаны.

В своей работе [43] Н. Жанг неоднократно показывает применение метода EMMS для расчета процесса горения в котле блока мощностью $150 \mathrm{MBT}$ Система реакций была упрощена горением углерода, а тепловой эффект испарения влаги и выхода летучих считался как средний теплоотвод, что повлияло на нижнюю часть топки.

В настоящее время опубликованы только несколько полуэмпирических моделей, которые позволяют решать ЦКС в трех измерениях. Одна из таких моделей разработана Т. Хуппаненом и позднее доработана K. Миоханеном в [44] (далее модель CFB3D). Другие аналогичные комплексные 3D-модели были представлены Р. Вишневским и Л. Ратсшоу в [45], а также Д. Палларесом в [46]. В этих моделях моделирование гидрогазодинамики упрощается, но описание химии горения представлено детальным образом вместе с различными неоднородными и однородными реакциями газификации и горения. Решение уравнения энергии, как правило, использует понятия, связанные с тепловым эффектом реакции, конвекцией газа и твердых частиц, теплопередачей к стенам и диффузионным перемешиванием в турбулентном потоке.

Палларес реализовал простую модель радиационного теплообмена в модели полуэмпирического процесса [46]. Лучистый теплообмен моделировался как серое излучение, в то время как перенос излучения между ячейками ограничивался тремя ортогональными направлениями граней ячейки для каждой шестигранной ячейки расчета. Влиянием излучающих газов пренебрегли. Модель была применена для моделирования промышленного образца котла с ЦКС мощностью 12 МВт, для которого подробных исходных данных не указано. Также сообщается, что в области с низкой концентрацией твердых частиц, таких как верхняя часть топки, количество тепла, выделенное в среду, существенно зависит от близлежащих областей [46]. Это доказывает потребность в более сложных излучающих моделях, которые учитывают эффект дальнего действия в данных видах систем.

Завершая вышеизложенный анализ, можно констатировать, что высокая трудоемкость натурных экспериментов, а в российской реальности иногда даже их недоступность, в сочетании с отсутствием обобщенных расчетных методик оставляет для разработки новых эффективных котлов с ЦКС путь использования надежного программного продукта для многопараметрического анализа конструктивных схем и соответствующих технологических решений.

\section{Метод Эйлера-Эйлера}

Как уже было подчеркнуто, необходимость решения большого количества взаимосвязанных дифференциальных уравнений, описывающих физикохимические процессы в камерах горения котельных агрегатов, относит их математическое моделирование к весьма сложным вычислениям. Поэтому от инженеров, занятых конструкторскими задачами, требуется высокая фундаментальная подготовка не только в области теории горения, тепло- и массообмена, гидрогазодинамики, но и дифференциального исчисления и вычислительной математики. На практике при решении инженерных задач это зачастую становится ограничивающим фактором для широкого использования методов математического моделирования. Ситуация стала меняться к лучшему, когда в последней четверти двадцатого века появились компьютерные программные продукты, доступные широкому кругу исследователей (например, зарубежные ANSYS Fluent \& CFX, отечественные FIRE 3D, $\sigma$-flou и др.).

В данном исследовании решение поставленных задач проведено с применением пакета прикладных программ Ansys v.12.1. Данный программный продукт непосредственно включает в себя расчетный модуль Fluent, позволяющий проводить численные исследования на основе встроенных или подключаемых математических моделей физических процессов. 
Первым шагом в решении любой многофазной задачи является определение того, какой из методов обеспечивает общие рекомендации к определению соответствующих моделей для каждого режима и степени межфазной связи для потоков, включающих пузырьки, капли или частицы, и соответствующую модель для различных количеств связи. Многофазные модели ЭЭ представлены в Ansys Fluent следующими моделями: моделью объема жидкости (VOF), модель смеси (The Mixture Model), моделю Эйлера (The Eulerian Model) [47].

Из перечисленных многофазных моделей ЭЭ модель представляет наибольшую сложность, поскольку в ней решаются уравнения импульса и неразрывности для каждой из сред из большого количества N уравнений. Взаимодействие достигается через давление и коэффициенты межфазного обмена. Способ, которым эта связь достигается, зависит от типа участвующих фаз: зернистые (жидкость-твердое тело) потоки обрабатываются иначе, чем негранулярные (жидкостьжидкость). Основные свойства гранулированных потоков приняты исходя из применения кинетической теории. Передача импульса при межфазовом взаимодействии зависит от математического описания среды. Пользовательские функции Ansys Fluent позволяют настроить расчет импульсного обмена [47].

В многофазной модели Эйлера фазы могут быть газами, жидкостями или твердыми телами в разнообразном сочетании. Она подходит для расчетов многофазных систем при взаимодействии фаз, в частности, таких как псевдоожиженные слои и суспензии частиц. Для каждой фазы используется Эйлеров подход, в отличие от ЭЛ подхода, который используется для дискретной фазовой модели. С многофазной моделью Эйлера количество вторичных фаз ограничено только требованиями к ресурсам вычислительной техники и требованиями сходимости. Можно смоделировать любое количество вторичных фаз при условии наличия достаточного объема памяти [47].

Как уже было отмечено, метод ЭЭ является одним из основных методов при моделировании котлов с ЦКС в научных исследованиях и промышленных масштабах. Для настоящего исследования в соответствии с рекомендациями также применена модель ЭЭ.

Сохранение массы, импульса и энергии записывается нижеследующими основными уравнениями.

Уравнение неразрывности для фазы $q$ :

$$
\frac{\partial}{\partial t}\left(\alpha_{q} \rho_{q}\right)+\nabla\left(\alpha_{q} \rho_{q} \vec{v}_{q}\right)=\sum_{p=1}^{n}\left(\dot{m}_{p q}-\dot{m}_{q p}\right)+S_{q},
$$

где $\vec{v}_{q}-$ скорость для фазы $q ; \dot{m}_{p q}-$ перенос массы от фазы $p$ до фазы $q ; \dot{m}_{q p}-$ перенос массы от фазы $q$ до фазы $p$. При этом источниковый член $S_{q}$ в правой части уравнения обычно равен нулю, но источник массы для каждой фазы можно задать постоянным или определяемым пользователем.

Уравнение баланса импульса для фазы $q$ :

$$
\frac{\partial}{\partial t}\left(\alpha_{q} \rho_{q} \vec{v}_{q}\right)+\nabla\left(\alpha_{q} \rho_{q} \vec{v}_{q} \vec{v}_{q}\right)=
$$

$$
\begin{aligned}
=-\alpha_{q} \nabla p+\nabla \bar{\tau}_{q}+ & \alpha_{q} \rho_{q} \vec{g}+\sum_{p=1}^{n}\left(\vec{R}_{p q}+\dot{m}_{p q} \vec{v}_{p q}-\dot{m}_{q p} \vec{v}_{q p}\right)+ \\
& +\left(\overrightarrow{F_{q}}+\overrightarrow{F_{l i f, q}}+\overrightarrow{F_{v m, q}}\right),
\end{aligned}
$$

$=$

где $\tau_{q}$ - тензор деформации напряжения фазы $q$.

$$
\stackrel{\overline{\tau_{q}}}{=} \alpha_{q} \mu_{q}\left(\nabla \overrightarrow{\boldsymbol{v}}_{q}+\nabla \overrightarrow{\boldsymbol{v}}_{q}^{T}\right)+\alpha_{q}\left(\lambda_{q}-\frac{2}{3} \mu_{q}\right) \nabla \overrightarrow{\boldsymbol{v}}_{q} \overline{\bar{I}},
$$

где $\mu_{q}$ и $\lambda_{q}$ - сдвиговая и объемная вязкость фазы $q$; $\overrightarrow{F_{q}}$ - сила внешнего тела; $\overrightarrow{F_{l i f t, q}}$ - подъемная сила; $\overrightarrow{F_{v m, q}}$ - виртуальная массовая сила; $\vec{R}_{p q}-$ сила взаимодействия между фазами; $p$ - давление, распределенное между фазами; $\vec{v}_{p q}-$ межфазовая скорость.

В многофазной модели Эйлера сохранение энергии записывается для каждой фазы отдельным уравнением энтальпий:

$$
\begin{gathered}
\frac{\partial}{\partial t}\left(\alpha_{q} \rho_{q} h_{q}\right)+\nabla\left(\alpha_{q} \rho_{q} \vec{u}_{q} h_{q}\right)=\alpha_{q} \frac{\partial p_{q}}{\partial t}+\bar{\tau}_{q}: \\
: \nabla \vec{u}_{q}-\nabla \vec{q}_{q}+S_{q}+\sum_{p=1}^{n}\left(Q_{p q}+\dot{m}_{p q} h_{p q}-\dot{m}_{q p} h_{q p}\right),
\end{gathered}
$$

где $h_{q}$ - удельная энтальпия $q$-й фазы; $\vec{q}_{q}-$ тепловой поток; $S_{q}-$ источниковый член уравнения, который включает источники энтальпии (например, из-за химической реакции или излучения); $Q_{p q}-$ интенсивность теплообмена между фазами $q$ и $p ; h_{p q}$ - межфазная энтальпия.

Кварцевый песок используется в большинстве опытов при проведении моделирования процессов в ЦКС, так как он подобен золе топлива по плотности и полидисперсности. Диапазон средних размеров составляет 0,1-0,3 мм при истинной плотности около

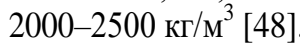

При определении влияния массы слоя и скорости газа характерная для режимов ЦКС масса слоя зависит от первоначальной засыпки, составляющей обычно 0,4-1 м. Насыпная плотность и гранулометрия слоя определяют перепад давлений в модели топки от 4 до 15 кПа, а диапазон скоростей газа на уровне от 3 до $7 \mathrm{M} / \mathrm{c}$ [46].

Для моделирования процессов в котлах с ЦКС важен корректный подход к подаче вторичного воздуха, расход которого составляет 30-60\% от всего расхода воздуха. При любой схеме ввода вторичного воздуха его взаимодействие с продуктами сгорания с аэродинамической точки зрения можно классифицировать как систему струй, внедряющихся в сносящий поток. При этом система имеет ряд особенностей, которые отличают ее от классических и в известной степени хорошо изученных такого рода течений [41]:

- высокое содержание взвешенных частиц в газовом потоке (50-100 кг/ $\mathrm{m}^{3}$ и более) на отметке ввода струй в топочный объем;

- изменение по высоте топки величины плотности и скорости сносящего потока;

- неравномерность полей плотности и скорости сносящего потока по сечению топки, соответ- 
ствующему плоскости ввода струй вторичного воздуха;

- организация процесса смешения, обеспечивающего догорание топливных частиц, вынесенных из плотного слоя, и доокисление продуктов химического недожога, образующегося в турбулентном слое при коэффициенте избытка первичного воздуха меньше единицы;

- обеспечение наряду с процессами догорания топлива условий для снижения генерации вредных веществ, и прежде всего $\mathrm{NO}_{\mathrm{x}}$;

- наличие условий для влияния струй вторичного воздуха на турбулентный слой вплоть до возникновения переменных полей концентрации частиц и скоростей газа на участке, прилегающем снизу к плоскости смешения [45].

В качестве геометрической модели для исследования была выбрана модель, представленная на рис. 2: топка с ЦКС, оборудованная двумя вводами топливных и инертных частиц на тыльной стене и десятью соплами вторичного дутья на фронтовой стене в два яруса. Данный котельный агрегат рассчитан с учетом установки двух циклонов на выходе из топочной камеры. Предполагается, что объем частиц, улавливаемых циклоном и вернувшихся в топку, равняется 90 \%. С учетом данного фактора вводится поправка на количество частиц, подаваемых в топку.

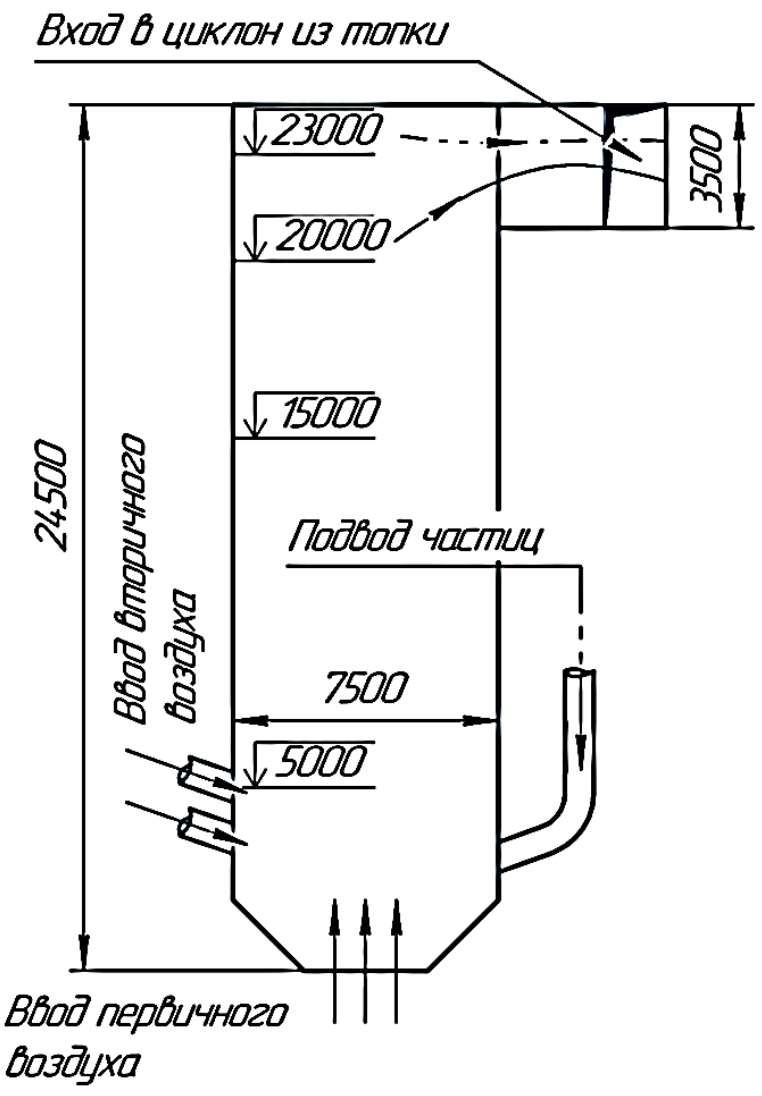

Рис. 2. Объект исследования

Fig. 2. Object of research
Предполагается, что моделирование не является реактивным и представляет собой систему псевдоожижения холодного потока, поэтому условия работы в данном случае определяются температурой 300 K и давлением 1 атм.

В процессе моделирования используются две фазы, которые поступают в топку через описание граничных и начальных условий входа и взаимодействуют посредством обмена массой и импульсом.

Газовая фаза представляется первичной. В процессе моделирования учитывает граничные условия входа и выхода потоков. Песок - вторичная фаза. Представляет локальный объем псевдоожиженного слоя. При моделировании песок представляется зернистым с постоянным размером 385 мкм и плотностью 2650 кг $\mathrm{M}^{3}$, принадлежа к группе частиц Гелдарт Б (Geldart B) [49-51].

В таблице приведен краткий список основных параметров, которые использовались при моделировании. Используемые параметры являются значениями, по умолчанию используемыми в Fluent, a также встречаются в работах других авторов [43-45] в качестве апробированных.

Таблица. Список используемых параметров с названием моделей

Table. $\quad$ List of used parameters with model name

\begin{tabular}{|c|c|}
\hline Параметр/Parameter & Модель/Model \\
\hline $\begin{array}{l}\text { Вязкость твердой частицы } \\
\text { Solid particle viscosity }\end{array}$ & $\begin{array}{l}\text { Гидаспов } \\
\text { Gidaspow }\end{array}$ \\
\hline $\begin{array}{l}\text { Насыпная вязкость твердой частицы } \\
\text { Solid particle bulk viscosity }\end{array}$ & $\begin{array}{l}\text { Лун и соавт. } \\
\text { Lun et al. }\end{array}$ \\
\hline $\begin{array}{l}\text { Фрикционная вязкость } \\
\text { Friction viscosity }\end{array}$ & $\begin{array}{l}\text { Шеффер } \\
\text { Sheffer }\end{array}$ \\
\hline \begin{tabular}{|l} 
Давление твердой частицы \\
Solid particle pressure
\end{tabular} & $\begin{array}{l}\text { Лун и соавт. } \\
\text { Lun et al. }\end{array}$ \\
\hline $\begin{array}{l}\text { Радиальная функция распределения } \\
\text { Radial distribution function }\end{array}$ & $\begin{array}{l}\text { Лун и соавт. } \\
\text { Lun et al. }\end{array}$ \\
\hline $\begin{array}{l}\text { Закон трения (газ - твердая частица) } \\
\text { Friction law (gas - solid particle) }\end{array}$ & $\begin{array}{l}\text { Гидаспов } \\
\text { Gidaspov }\end{array}$ \\
\hline $\begin{array}{l}\text { Закон трения (твердая частица - твердая } \\
\text { частица) } \\
\text { Friction law (solid particle - solid particle) }\end{array}$ & $\begin{array}{l}\text { Сиамлал-O'Брайен } \\
\text { Syamlal-O’Brien }\end{array}$ \\
\hline
\end{tabular}

На рис. 3 представлен контурный график распределения частиц песка (кг/кг) по высоте топки. Наблюдается повышенное содержание частиц в нижней части топки, поэтому принципиальным является результат взаимодействия частиц между собой, а также внутренняя циркуляция дисперсного материала. В средней части топки наблюдается пристенное опускное течение, интенсивность которого уменьшается с высотой. Низкая концентрация твердой фазы вверху топки, как показано в [48], соответствует течению двухфазной среды в режиме пневмотранспорта.

На рис. 4 отображены контурные графики скорости частиц песка, где наблюдается следующая зависимость - увеличение скорости от низа (циркулирующий слой) к верху (выход в циклоны). Средняя скорость по сечениям топочной камеры $7 \mathrm{~m} / \mathrm{c}$. 

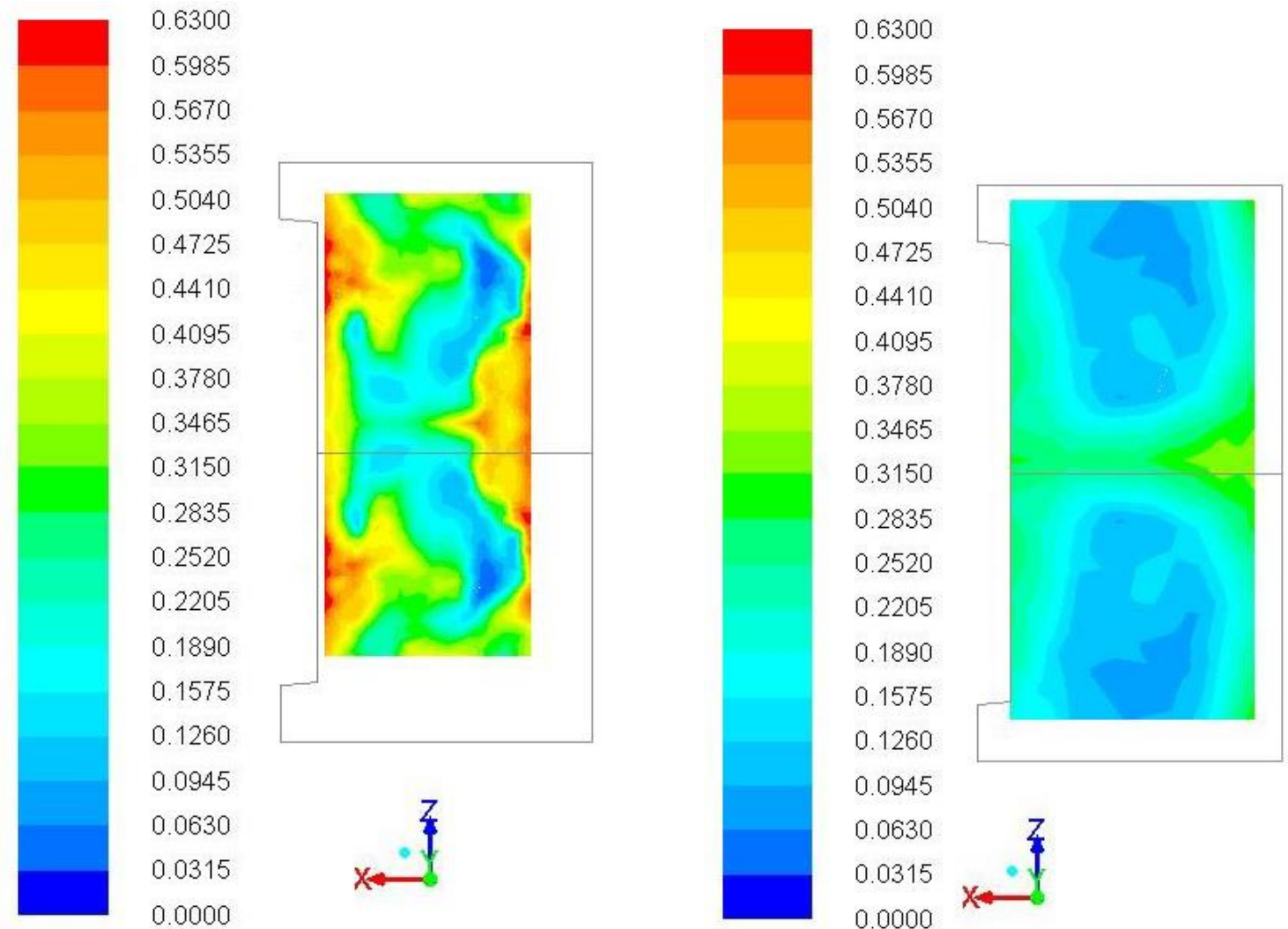

$a / a$

0.0000

$\sigma / b$

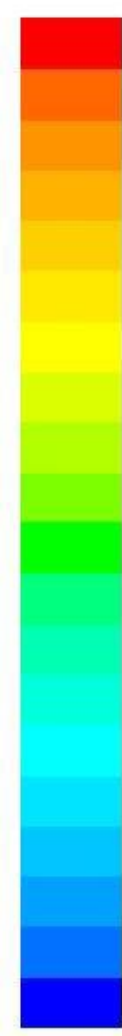

0.6300

0.5985

0.5670

0.5355

0.5040

0.4725

0.4410

0.4095

0.3780

0.3465

0.3150

0.2835

0.2520

0.2205

0.1890

0.1575

0.1260

0.0945

0.0630

0.0315

0.0000

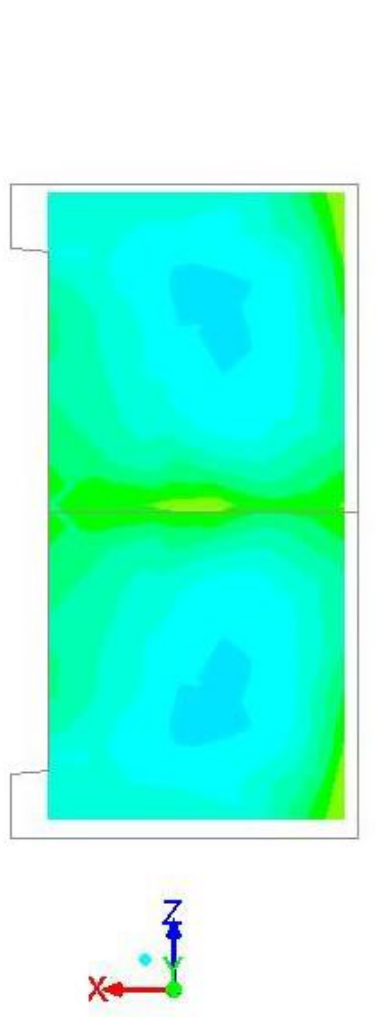

b/c

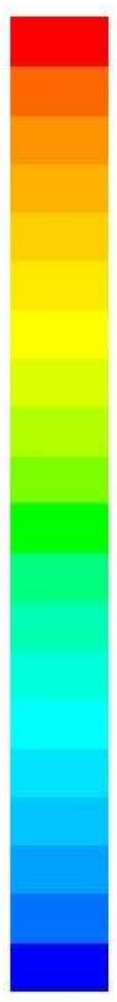

0.6300

0.5985

0.5670

0.5355

0.5040

0.4725

0.4410

0.4095

0.3780

0.3465

0.3150

0.2835

0.2520

0.2205

0.1890

0.1575

0.1260

0.0945

0.0630

0.0315

0.0000

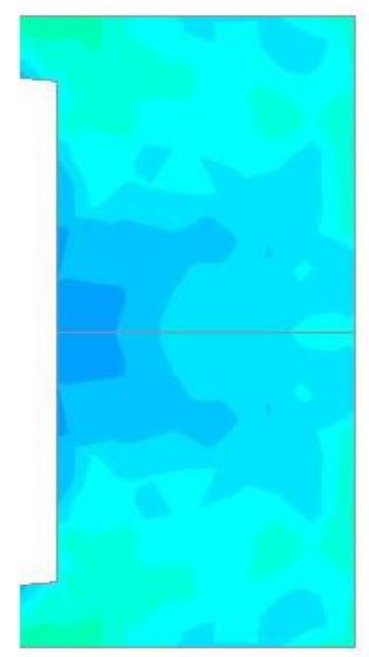

Pис. 3. Контурный график распределения частии песка (кг/кг): а-г) распределение в горизонтальных плоскостях (5, 15, 20 и 23 метров по высоте топки соответственно)

Fig. 3. Contours of sand particles distribution ( $\mathrm{kg} / \mathrm{kg}): a-d)$ distribution in horizontal planes (5, 15, 20 and 23 meters in height of the furnace, respectively) 


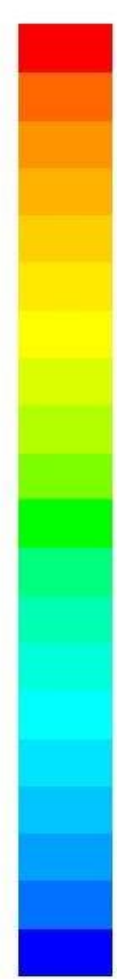

30.0000

28.5000

27.0000

25.5000

24.0000

22.5000

21.0000

19.5000

18.0000

16.5000

15.0000

13.5000

12.0000

10.5000

9.0000

7.5000

6.0000

4.5000

3.0000

1.5000

0.0000

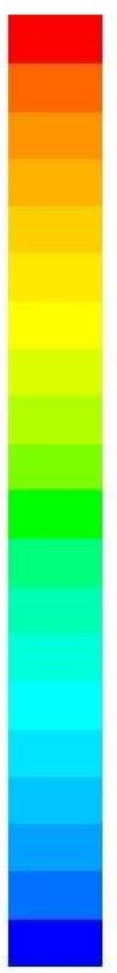

30.0000

28.5000

27.0000

25.5000

24.0000

22.5000

21.0000

19.5000

18.0000

16.5000

15.0000

13.5000

12.0000

10.5000

9.0000

7.5000

6.0000

4.5000

3.0000

1.5000

0.0000
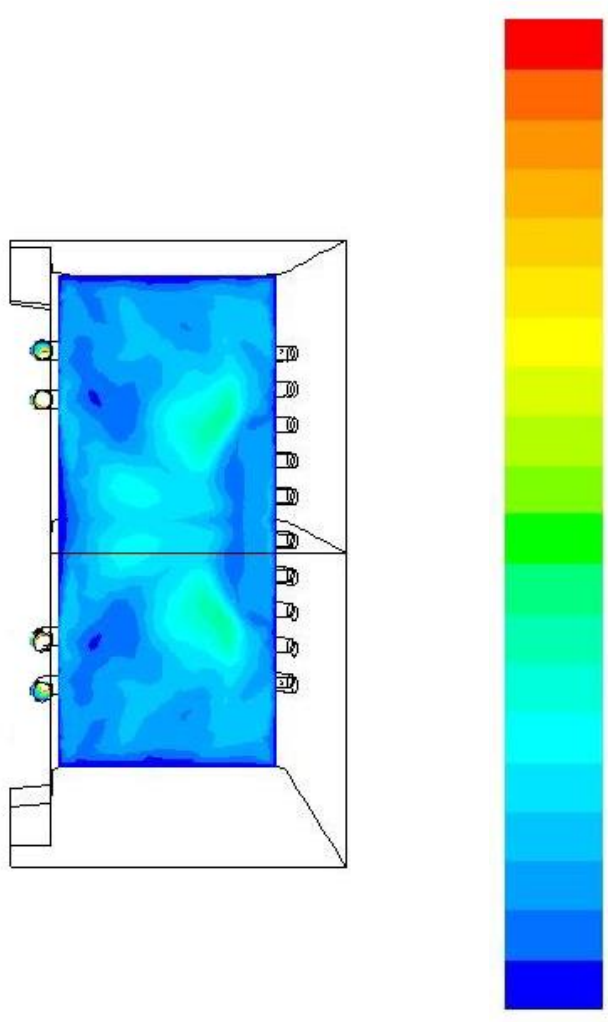

$a / a$
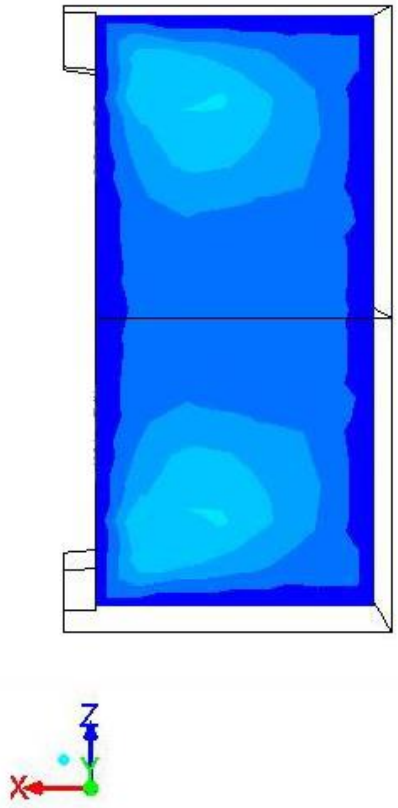

b/c
30.0000

28.5000

27.0000

25.5000

24.0000

22.5000

21.0000

19.5000

18.0000

16.5000

15.0000

13.5000

12.0000

10.5000

9.0000

7.5000

6.0000

4.5000

3.0000

1.5000

0.0000
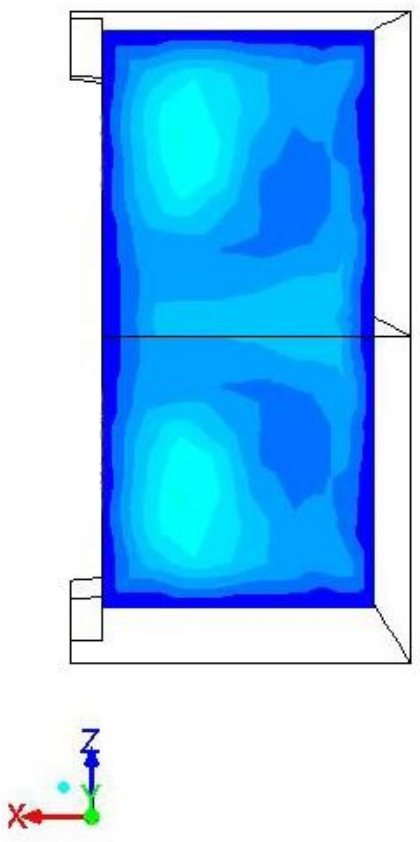

$\sigma / b$

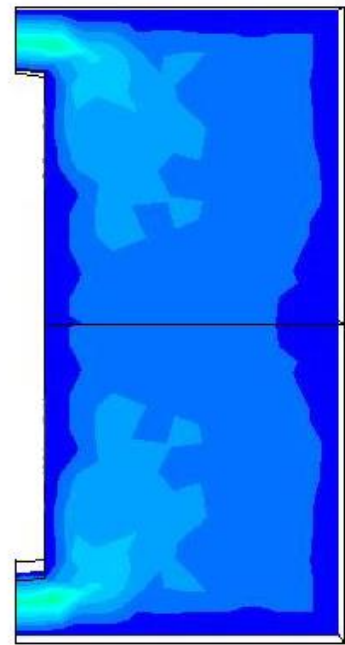

6.0000

4.5000

3.0000

1.5000

0.0000

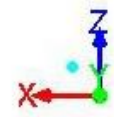

$2 / d$

Pис. 4. Контурный график распределения скорости частии песка по объему топки с циркулирующим кипящим слоем $($ м/c): a-г) распределение в горизонтальных плоскостях $(5,15,20$ и 23 метра по высоте топки соответственно)

Fig. 4. Contours of sand particles velocity distribution over the furnace volume with circulating fluidized bed $(\mathrm{m} / \mathrm{s}): a-d)$ distribution in horizontal planes (5, 15, 20 and 23 meters in height of the furnace, respectively) 


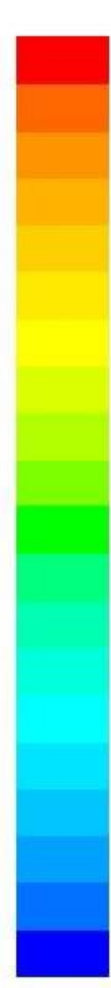

30.0000
28.5000
27.0000
25.5000
24.0000
22.5000
21.0000
19.5000
18.0000
16.5000
15.0000
13.5000
12.0000
10.5000
9.0000
7.5000
6.0000
4.5000
3.0000
1.5000
0.0000

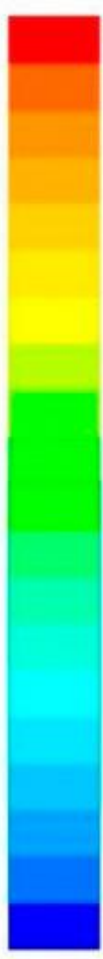

30.0000

28.5000

27.0000

25.5000

24,0000

22.5000

21.0000

19.5000

18.0000

16.5000

15.0000

13.5000

12.0000

10.5000

9.0000

7.5000

6.0000

4.5000

3.0000

1.5000

0.0000

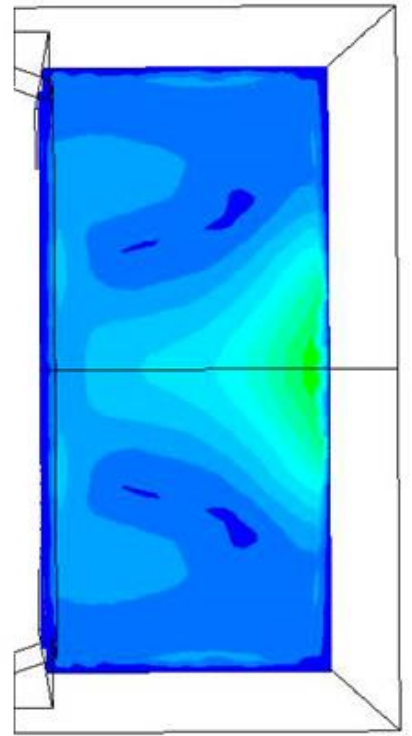

$a / a$

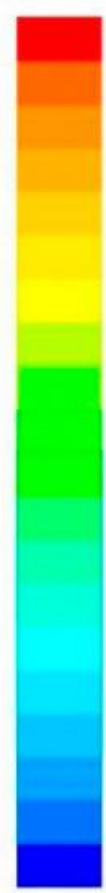
30.0000
28.5000
27.0000
25.5000
24.0000
22.5000
21.0000
19.5000
18.0000
16.5000
15.0000
13.5000
12.0000
10.5000
9.0000
7.5000
6.0000
4.5000
3.0000
1.5000
0.0000
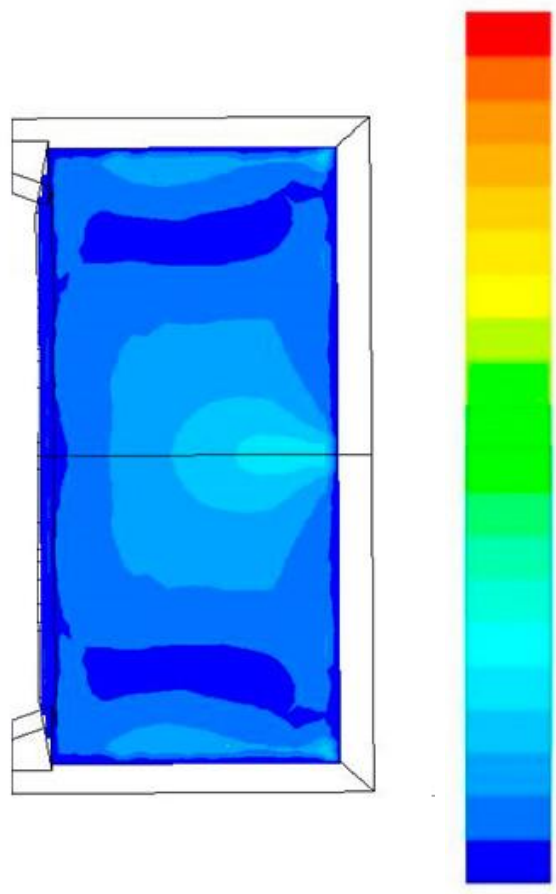

30.0000

28.5000

27.0000

25.5000

24.0000

22.5000

21.0000

19.5000

18.0000

16.5000

15.0000

13.5000

12.0000

10.5000

9.0000

7.5000

6.0000

4.5000

3.0000

1.5000

0.0000

b/c

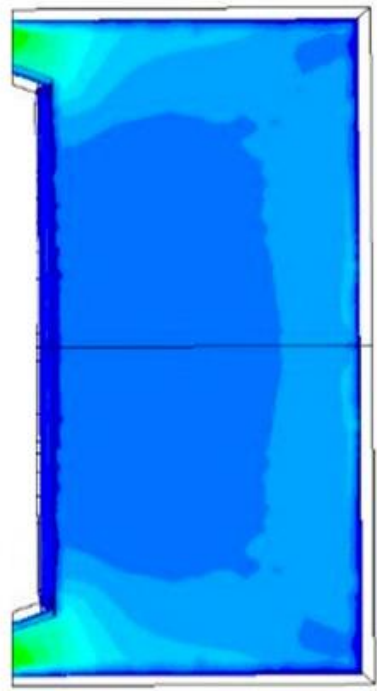

2/d

Pис. 5. Контурный график распределения скорости частии песка по объему топки с ЦКС (м/c): а-г) распределение в горизонтальных плоскостях (5, 15, 20 и 23 метров по высоте топки соответственно)

Fig. 5. Contours of sand particles velocity distribution over the furnace volume with $C F B(\mathrm{~m} / \mathrm{s})$ : $a-d)$ distribution in horizontal planes $(5,15,20$ and 23 meters in height of the furnace, respectively)

\section{Метод Эйлера-Лагранжа}

В методе ЭЛ уравнения сохранения решаются для непрерывной фазы, а фаза частиц отслеживается путем решения уравнений движения для каждой частицы.
Уравнения непрерывности, импульса, энергии и переноса частиц для газовой фазы представлены уравнениями: 


$$
\begin{gathered}
\frac{\partial}{\partial t}\left(\varepsilon_{f} \rho_{f}\right)+\nabla\left(\varepsilon_{f} \rho_{f} \vec{v}_{f}\right)=S_{\text {mas }} ; \\
\frac{\partial}{\partial t}\left(\varepsilon_{f} \rho_{f}\right)+\nabla\left(\varepsilon_{f} \rho_{f} \vec{v}_{f} \vec{v}_{f}\right)= \\
=-\varepsilon_{f} \nabla p+\nabla \tau_{f}+\varepsilon_{f} \rho_{f} g+K_{D P M}\left(\vec{v}_{s}-\vec{v}_{f}\right)+S_{m o m} ; \\
\frac{\partial}{\partial t}\left(\varepsilon_{f} \rho_{f} h_{f}\right)+\nabla\left(\varepsilon_{f} \rho_{f} \vec{v}_{f} h_{f}\right)=-\varepsilon_{f} \frac{\partial p}{\partial t}+\tau_{f}: \\
: \nabla \vec{v}_{f}-\nabla \vec{q}_{f}-\nabla\left[\varepsilon_{f} \sum_{k=1}^{m} h_{f, k} \overrightarrow{J_{k}}\right]+S_{f, r a d}+S_{f, r e a}+S_{e n} ; \\
\frac{\partial}{\partial t}\left(\varepsilon_{f} \rho_{f} Y_{f, k}\right)+\nabla\left(\varepsilon_{f} \rho_{f} \vec{v}_{f} Y_{f, k}\right)=\nabla \varepsilon_{f} \overrightarrow{J_{K}}+\varepsilon_{f} R_{f, k}+S_{s p},
\end{gathered}
$$

где $\rho$ - плотность; $\varepsilon$ - объемная доля; $\vec{v}$ - вектор скорости; $p$ - давление; $\tau_{f}-$ тензор касательных напряжений; $g$ - вектор ускорения свободного падения; $s$ - индекс твердой фазы; $K_{D P M}$ - коэффициент межфазного обмена в результате сопротивления, рассчитанный для объемной доли твердых частиц в Эйлеровой сетке; $h$ удельная энтальпия; $k$ - индекс частиц; $Y_{f, k}-$ массовая доля частицы $k ; m$ - число частиц в газовой фазе; $\overrightarrow{J_{k}}$ диффузионный поток частиц $k ; q$ - тепловой поток. Исходный термин $S_{f, r a d}$ составляет вклад в уравнение энергии за счет излучения, $S_{f, r e a}$ определяет энергию, выделяемую в гомогенных реакциях. $R_{f, k}$ представляет собой темп образования однородных частиц $k$. $S_{\text {mas }}$, $S_{\text {mom }}, S_{e n}, S_{s p}$ - коэффициенты, вызванные обменом массы, импульсом, энергии и частиц между непрерывной и дискретной фазами соответственно.

Расчетная скорость ожижения при реальных физических параметрах эксплуатации котла с ЦКС (температура слоя $850^{\circ} \mathrm{C}$ ) составляет $4,6 \mathrm{~m} / \mathrm{c}$, однако максимальная скорость в топке по расчетам принимается с $50 \%$ запасом и равна 7 м/с [52]. Как видно из рис. 5 , скорость среды, определенная по сечению топочной камеры, составляет 7 м/с. На выходе из топки она возрастает до $20 \mathrm{~m} / \mathrm{c}$.

\section{Анализ полученных результатов}

Для сравнения двух выбранных методов (ЭЭ и ЭЛ) оценкой на сходимость результатов моделирования аэродинамики топки котла с ЦКС построен график распределения скорости потока воздуха по высоте топочной камеры (рис. 6). Математическое усреднение показаний произведено в программе Microsoft Excel линией тренда. По графику видно линейное увеличение скорости на всем интервале увеличения высоты для обоих методов. Максимальная скорость проявляется в области выходного окна из топки. Также на графике наблюдается участок падения скоростей при достижении высоты 5-15 м в области циркуляции частиц, когда недогоревшие частицы возвращаются обратно в слой в связи с увеличением сопротивления, за счет взаимного столкновения частиц и контакта со стенами топочной камеры, а также из-за недостатка кинетической энергии для выноса крупных частиц. В данном исследовании возврат частиц связан лишь с увеличением сопротивления на данной высоте. Согласно контурным графикам рис. 3-5 можно сделать заключение о том, что в области циркуляции недогоревшие частицы возвращаются вниз преимущественно в околостенных зонах. На основании этого можно прогнозировать условия надежности в эксплуатации топочных экранов. Согласно статистике [53] одна из часто выводимых из работы систем в котлах с ЦКС - это оборудование, находящееся под давлением.

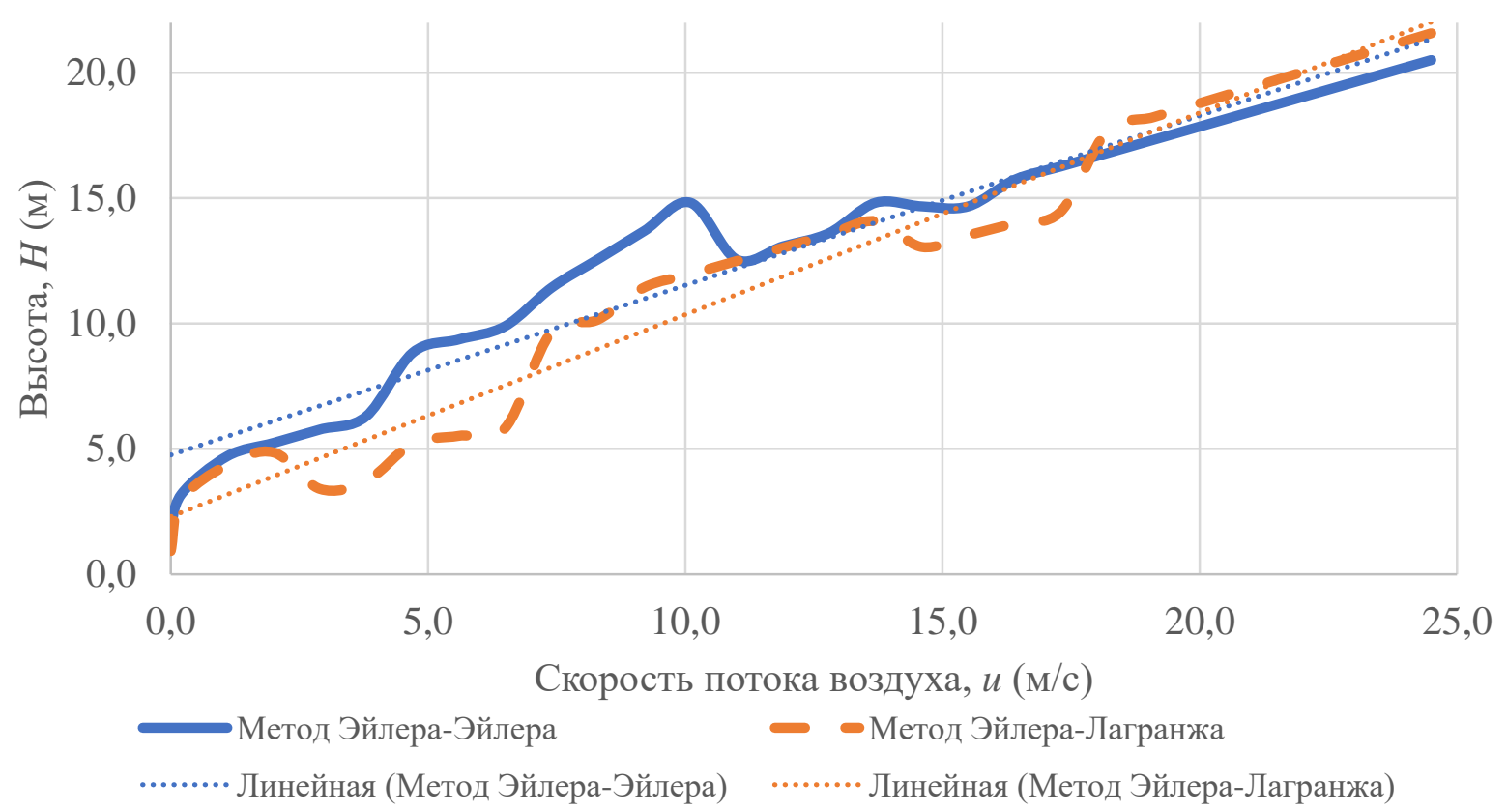

Рис. 6. График распределения скорости потока воздуха по высоте топочной камеры по методам Эйлера-Эйлера и Эйлера-Лагранжа

Fig. 6. Graph of air flow rate distribution by the combustion chamber height according to the Euler-Euler and EulerLagrange methods 
В связи с большим накоплением частиц возможны налипания, которые приводят к непроектным режимам в части теплопередачи и дальнейшим разрывам труб. Поэтому необходимо размещать устья вторичного воздуха по высоте топки таким образом, чтобы развивались внутритопочные течения, которые будут располагать к надежной гидродинамике.

\section{Заключение}

Псевдоожижение является хорошо зарекомендованной технологией, используемой во многих промышленных процессах. Однако точное моделирование сложных явлений, происходящих в системах с псевдоожиженным слоем, по-прежнему является большой проблемой как для ученых, так и для инженеров. Исходя из изложенного в статье обзора различных подходов к моделированию, используемых для псевдоожиженного слоя, можно сделать вывод о том, что псевдоожижение возможно смоделировать на различных уровнях детализации. В зависимости от того, насколько детально требуется описать мультифазный поток, необходимо применять соответствующие стратегии.

Например, при моделировании методом Computational Fluid Dynamics используется уравнение Эргуна, так как пустотность всегда меньше 0,8 (значение, предложенное Д. Гидасповым). Однако уравнение Вена и Ю дает более точный результат и должно использоваться вместо него. Поэтому стараются использовать данные корреляции совместно. Тем не менее такие выводы не могут быть обобщены на все системы, необходимо рассматривать каждый случай отдельно. Все сводится к тому, чтобы понимать, когда необходимо применять то или иное уравнение.

В настоящей работе методы Эйлера-Эйлера и Эйлера-Лагранжа приведены в формате тестового сравнения, что дает представление и о их взаимном применении в зависимости от цели моделирования. В ходе численного исследования решались задачи приведения геометрических размеров, граничных условий и методов в соответствие к тем, которые приводятся в литературных источниках в качестве апробированных.

Для расчета аэродинамики исследуемого объекта был применен метод Эйлера-Эйлера. Однако данный метод имеет ряд недостатков, например, отсутствие пост-анализа по распределению частиц по диаметрам

\section{СПИСОК ЛИТЕРАТУРЫ}

1. Research trends and recent advances of oxyfuel combustion of solid fuels in fluidized beds / Q. Liu, W. Zhong, Y. Shao, A. Yu // CIESC Journal. - 2019. - V. 3. - P. 1-23

2. Распоряжение Правительства Российской Федерации № 1715-р «Энергетическая стратегия России на период до 2030 года» от 13 ноября 2009 г // Министерство Энергетики РФ. URL: https://minenergo.gov.ru/node/1026 (дата обращения 12.11.2019).

3. Myöhänen K. Modelling of combustion and sorbent reactions in three-dimensional flow environment of a circulating fluidized bed furnace: Dr. Sc. Diss. - Lappeenranta, 2011. - 164 p.

4. The role of mesoscale structures in rapid gas-solid flow K. Agrawal, P.N. Loezos, M. Syamlal, S. Sundaresan // Journal of Fluid Mechanics. - 2001. - V. 445. - P. 151-185. в исследуемом объекте (отсюда необходимость в задании нескольких фракций), а также отсутствие задания условий горения угольных частиц. В результате моделирования по методу Эйлера-Эйлера были получены контурные графики распределения частиц и скорости по высоте топки. График распределения частиц песка схож с тем, что представлен в [44], соответствуя особенностям функционирования в топочном устройстве с циркулирующим кипящим слоем, к которым относится неравномерное распределение концентрации твердых частиц по высоте топки. Наблюдается высокая концентрация частиц в нижней части топки, которая уменьшается с высотой и распределяется в околостенных зонах.

В подходе Эйлера-Лагранжа частицы моделируются как дискретные объекты с индивидуальными диаметрами. Данный метод находит редкое применении при моделировании объектов промышленного назначения, так как требователен к вычислительным затратам и наличию значительного временного запаса. Эти факторы негативно отражаются на исследованиях, в особенности на начальных этапах расчета, когда имеет важное значение скорость анализа влияния применяемых конструктивных решений. Также метод применяется только в сильно разбавленных системах с небольшим числом частиц и требует усреднения значений, полученных с помощью математического моделирования для получения качественных результатов. Изменение значений скорости потоков в сечении топочной камеры по методу Эйлера-Лагранжа совпадает с методом Эйлера-Эйлера и равняется 7 м/с.

Обсуждение выделенных в настоящей работе ключевых результатов, полученных в ходе моделирования по методу Эйлера-Эйлера и Эйлера-Лагранжа топки с ЦКС, подчеркивает последующие задачи. В частности, для дальнейшей проработки вопросов моделирования топок с циркулирующим кипящим слоем целесообразно изучать по методу ЭйлераЭйлера закономерности выноса твердой фазы из плотного слоя в зависимости от скорости псевдоожижения, расположения и взаимной работы устьев ввода вторичного воздуха по высоте топки, а также от добавления системы возврата в нижнюю часть топки для накопления частиц.

Исследование выполнено при финансовой поддержке РФФИ (проект No. 18-38-00775_мол_а).

5. Reh L. Development potentials and research needs in circulating fluidized bed combustion // China Particuology. - 2003. - V. 1. № 5. - P. 185-200.

6. Comparison of multifluid and discrete particle modelling in numerical predictions of gas particle flow in circulating fluidised beds / C.H. Ibsen, E. Helland, B.H. Hjertager, T. Solberg, L. Tadrist, R. Occelli // Powder Technology. - 2004. - V. 149. № 1. - P. 29-41.

7. Andrews A.T., Loezos P.N., Sundaresan S. Coarse-grid simulation of gas-particle flows in vertical risers// Industrial \& Engineering Chemistry Research. - 2005. - V. 44. - P. 6022-6037.

8. Kallio S. Comparison of simulated and measured voidage and velocity profiles and fluctuations in a CFB riser // Proc. of the $8^{\text {th }}$ International Conference on Circulating Fluidized Beds. Hangzhou, China, 2005. - P. 105-112. 
9. Filtered two-fluid models for fluidized gas-particle suspensions / Y. Igci, A.T. Andrews, S. Sundaresan, S. Pannala, T. O'Brien // AIChe Journal. - 2008. - V. 54. - № 6. - P. 1431-1448. URL: https://doi.org/10.1002/aic.11481 (дата обращения 10.02.2020).

10. CFD-DEM study of the effect of cyclone arrangements on the gassolid flow dynamics in the full-loop circulating fluidized bed S. Wang, K. Luo, C. Hu, J. Fan // Chemical Engineering Science. - 2017. - V. 172. - P. 199-215.

11. Tu Q., Wang H. CPFD study of a full-loop three-dimensional pilotscale circulating fluidized bed based on EMMS drag model // Powder Technology. - 2018. - V. 323. - P. 534-547.

12. Crowe C., Sommerfeld M., Tsuji Y. Multiphase flows with droplets and particles. - Boca Raton: CRC Press, 1998. - 496 p.

13. Review of discrete particle modeling of fluidized beds / N.G. Deen M. van Sint Annaland, M.A. van der Hoef, J.A.M. Kuipers // Chemical Engineering Science. - 2008. - V. 62 - P. 28-44.

14. Numerical investigation of particle transport hydrodynamics and coal combustion in an industrial-scale circulating fluidized bed combustor: effects of coal feeder positions and coal feeding rates / M. Massoudi Farid, H.J. Jeong, K.H. Kim, J. Lee, D. Kim, J. Hwang // Fuel. - 2017. - V. 192. - P. 187-200.

15. Numerical simulation of circulating fluidized bed oxy-fuel combustion with Dense Discrete Phase Model / Y. Wu, D. Liu, D. Zheng, J. Ma, L. Duan, X. Chen // Fuel Processing Technology. - 2019. - V. 195. - P. 1-14.

16. Goldschmidt M.J.V. Hydrodynamic modelling of fluidised bed spray granulation. Doctoral dissertation. - Netherlands, 2001. $303 \mathrm{p}$.

17. A model for improving the Euler-Euler twophase flow theory to predict chemical reactions in circulating fluidized beds / B. Hou, X. Wang, T. Zhang, H. Li // Powder Technology. -2017. V. 321. - P. 13-30.

18. Improved numerical approaches to predict hydrodynamics in a pilot-scale bubbling fluidized bed biomass reactor: a numerica study with experimental validation / J. Cardoso, V. Silva, D. Eusébio, P. Brito, L. Tarelho // Energy Conversion and Management. - 2018. - V. 156. - P. 53-67.

19. Kallio S., Kilpinen P., Hupa M. Advanced modeling of nitrogen oxide emissions in circulating fluidized bed combustors: parametric study of hydrodynamics $/ / 6^{\text {th }}$ Int. Conference on Circulating Fluidized Beds. - Würzburg, 1999. - P. 659-664.

20. Kilpinen P., Kallio S., Hupa M. Advanced modeling of nitrogen oxide emissions in CFBC: parametric study of coal combustion and nitrogen compound chemistries $/ /$ Proc. of the $15^{\text {th }}$ International Conference on FBC. - Savannah, GA, USA, 1999. Paper No. FBC99-0155.

21. Chapman S., Cowling T. G., Burnett D. The mathematical theory of non-uniform gases: an account of the kinetic theory of viscosity, thermal conduction and diffusion in gases. - Cambridge: Cambridge university press, $1990 .-423 \mathrm{p}$.

22. Simulation of particles and gas flow behavior in the riser section of a circulating fluidized bed using the kinetic theory approach for the particulate phase / S. Benyahia, H. Arastoopour, T.M. Knowlton, H. Massah // Powder Technology. - 2000. V. 112. - № 1-2. - P. 24-33.

23. Bird R.B., Stewart W.E., Lightfoot E.N. Transport phenomena $\left(2^{\text {nd }}\right.$ ed.). - New York: Wiley, 2002. $-885 \mathrm{p}$.

24. Taghipour F., Ellis N., Wong C. Experimental and computational study of gas-solid fluidized bed hydrodynamics // Chemical Engineering Science. - 2005. - V. 60. - P. 6857-6867.

25. Ergun S. Fluid flow through packed columns // Chemical Engineering Progress. - 1952. - V. 48. - P. 89-94.

26. Wen C.Y., Yu Y.H. Mechanics of fluidization // Chemical Engineering Progress Symposium Series. - 1966. - V. 62. P. $100-111$.

27. Eulerian simulation of gas-solid two-phase flow in a CFB-riser under consideration of cluster effects / H. Qi, C. You, A. Boemer, U. Renz // Fluidization 2000: Science and Technology / Eds. D. Xu, S. Mori. - Xi'an: Xi'an Publ. House, 2000 - P. 231-237.

28. Numerical study of segregation using a new drag force correlation for polydisperse systems derived from lattice-Boltzmann simulations / R. Beetstra, M.A. van der Hoef, J.A.M. Kuipers // Chemical Engineering Science. - 2007. - V. 62. - № 1-2. P. 246-255.
29. CFD simulation of concurrent upgas solidflow in circulating fluidized beds with structure-dependent drag coefficient / N. Yang, W. Wang, W. Ge, J. Li // Chemical Engineering Journal. - 2003. V. 96. - P. 71-80.

30. Modeling of drag with the Eulerian approachand EMMS theory for heterogeneous dense gas-solid two-phase flow / H. Qi, F. Li, B. Xi, C. You // Chemical Engineering Science. - 2007. - V. 62. P. $1670-1681$.

31. Li J., Kwauk M. Particle-fluid two-phase flow: the energyminimization multi-scale method. - Beijing: Metallurgical Industry Press, 1994. $-215 \mathrm{p}$.

32. Guangbo Z. A coal combustion model for circulating fluidized bed boilers // Fuel. - 2000. - V. 79. - P. 165-172.

33. Gungor A. Hydrodynamic modeling of a circulating fluidized bed // Powder Technology. - 2007. - V. 172. - P. 1-13.

34. Nakamura $\mathrm{H}$. Numerical modeling of particle fluidization behavior in a rotating fluidized bed // Powder Technology. - 2007. V. 171. - P. 106-117.

35. Hadavand A. An innovative bed temperature-oriented modeling and robust control of a circulating fluidized bed combustor // Chemical Engineering Journal. - 2008. - V. 140. - P. 497-508.

36. Kong L.X., Hodgson P.D. Computational simulation of gas flow and heat transfer near an immersed object in fluidized beds // Advances in Engineering Software. - 2007. - V. 38. - P. 826-834.

37. Bastos J.C.S.C. Modelling and simulation of a gas-solids dispersion flow in a high flux circulating fluidized bed (HFCFB) riser // Catalysis Today. - 2008. - V. 130 - P. 462-470.

38. Cornelissen J.T. CFD modelling of a liquid-solid fluidized bed // Chemical Engineering Science. - 2007. - V. 62. - P. 6334-6348.

39. Modeling of particle transport and combustion phenomena in a large-scale circulating fluidized bed boiler using a hybrid EulerLagrange approach / W.P. Adamczyk, G. Wecel, M. Klajny, P. Kozołub, A. Klimanek, R.A. Białecki // Particuology. - 2014. V. 16. - P. 29-40.

40. Bordbar M.H., Myohanen K., Hyppanen T. Coupling of a radiative heat transfer model and a three-dimensional combustion model for a circulating fluidized bed furnace // Applied Thermal Engineering. - 2015. - V. 76. - P. 344-356.

41. Bordbar M.H., Wecel G., Hyppanen T. A line by line based weighted sum of gray gases model for inhomogeneous $\mathrm{CO}_{2}-\mathrm{H}_{2} \mathrm{O}$ mixture in oxy-fired combustion // Combustion and Flame. 2014. - V. 161. - P. 2435-2445.

42. Nies M., Plackmeyer J. Computer-aided optimisation of gasparticle flow and combustion at the duisburg circulating fluidised bed furnace // VGB PowerTech Journal. - 2011. - V. 8. - P. 64-69.

43. Zhang N., Wang W., Li J. CFD simulation of combustion in a 150 MWe circulating fluidized bed boiler // Cleaner Combustion and Sustainable World - Proc. of the $7^{\text {th }}$ International Symposium on Coal Combustion / Eds. H. Qi, B. Zhao. - Berlin: SpringerVerlag, 2013. - P. 65-67.

44. Myöhänen K., Hyppänen T. A three-dimensional model frame for modelling combustion and gasification in circulating fluidized bed furnaces // International Journal of Chemical Reactor Engineering. - 2011. - V. 9. - № 1. - P. 1-55.

45. Reactive gas-solids flows in large volumes - 3D modeling of industrial circulating fluidized bed combustors / R. Wischnewski, L. Ratschow, E.U. Hartge, J. Werther // Particulogy. - 2010. V. 8. - P. 67-77.

46. Modeling of the heat transfer in large-scale circulating fluidized bed furnaces / D. Pallares, M. Palonen, V. Yla-Outinen, F. Johnsson // Proc. of the $21^{\text {st }}$ International Conference on Fluidized Bed Combustion / Eds. U. Arena, R. Chirone, M. Miccio, P. Salatino. - EnzoAlbanoEditore, Naples, 2012. - P. 867-874.

47. ANSYS FLUENT 12.0, Theory Guide. 2009.

URL: https://www.afs.enea.it/project/neptunius/docs/fluent/html/th /main_pre.htm (дата обращения 12.11.2019).

48. Численное исследование влияния дисперсного состава на характеристики сжигания угольного топлива в циркулирующем кипящем слое / В.В. Саломатов, А.В. Гиль, А.В. Старченко, Р.Ю. Архипов // Вестник Новосибирского государственного университета. Серия: Физика. - 2016. - Т. 11. № 3. - C. 53-61.

49. Modelling of particle flow in a dual circulation fluidized bed by a Eulerian-Lagrangian approach / J. Gu, Y. Shao, X. Liu, W. Zhong, 
A. Yu // Chemical Engineering Science. - 2018. - V. 192. P. 619-633.

50. Experimental study and CFD modelling of minimum fluidization velocity for Geldart A, B and D particles / S. Amarasinghe, K. Jayarathna, S. Ahangama, M.E. Moldestad, L.-A. Tokheim // International Journal of Modeling and Optimization. - 2017. V. 7. - P. $152-156$.

51. Bandara J.C., Eikeland M.S., Moldestad B.M.E. Analyzing the effects of particle density, size, size distribution and shape for minimum fluidization velocity with Eulerian-Lagrangian, CFD simulation // Proc. of the $58^{\text {th }}$ Conference on Simulation and Modelling. - Reykjavik, Iceland, 2017. - P. 60-65.

52. Кокшарев О.М. Анализ применимости численных алгоритмов для моделирования процессов в топке котла с циркулирующим кипящим слоем: магистерская диссертация. - Томск, 2017. $-125 \mathrm{c}$.

53. Suik H., Pihu T. Warranty reliability of CFB boiler burning oil shale // Oil Shale. - 2009. - V. 26. - № 2. - P. 99-107.

Поступила 22.02.2020 2.

\section{Информация об авторах}

Гиль A.B., кандидат технических наук, доцент научно-образовательного центра И.Н. Бутакова, Национальный исследовательский Томский политехнический университет.

Заворин А.C., доктор технических наук, профессор, руководитель научно-образовательного центра И.Н. Бутакова, Национальный исследовательский Томский политехнический университет.

Кокшарев О.М., аспирант научно-образовательного центра И.Н. Бутакова, Национальный исследовательский Томский политехнический университет. 
UDC 621.18

\title{
APPROACHES TO COMPUTATIONAL STUDY OF COMBUSTION CHAMBERS WITH CIRCULATING FLUIDIZED BED
}

Andrey V. Gil, angil@tpu.ru

\author{
Alexander S. Zavorin, \\ kotel@tpu.ru \\ Oleg M. Koksharev, \\ omk2@tpu.ru \\ 1 National Research Tomsk Polytechnic University, \\ 30, Lenin avenue, Tomsk, 634050, Russia.
}

The relevance. In modern scientific research the computing experiment is one of the most productive means of studying complex tasks where the outcome depends on simultaneous influence of aerodynamics, heat transfer and combustion. Numerical calculations allow not only explaining correctly the physical phenomena noted at experimental installations, but quite often considerably extending expensive and labor-consuming physical or full-scale experiment with computer simulation. In this regard, the relevance of the research aimed at improving the methods of numerical analysis of furnace processes in boilers with circulating fluidized bed on the basis of computer mathematical modeling is very high to justify the basic design and technological decisions taken when creating boilers with circulating fluidized bed. In addition to practical significance, the research has a high scientific component, since the fundamental laws of physical and chemical processes in the circulating fluidized bed are the basis of the task.

The aim of the research is to determine the approaches and applicability of numerical algorithms with satisfactory level of detail and convergence for modeling furnace processes in boilers with circulating fluidized bed.

Object: furnace with circulating fluidized bed, equipped with two inputs of fuel and inert particles on the rear wall and ten secondary air inlets on the front wall in two decks.

Methods: mathematical modeling of physical and chemical processes in a furnace chamber with circulating fluidized bed based on EulerEuler and Euler-Lagrangian approaches using RANS models. Differential equations for conservation of mass, momentum, energy and particle transport are written. The numerical study was carried out using the Ansys Fluent V. 12.1 software package.

Result. The initial and boundary conditions for numerical simulation of processes in the furnace chamber of a boiler unit with circulating fluidized bed using Euler-Euler and Euler-Lagrangian approaches are determined.

\section{Key word:}

Circulating fluidized bed boiler, mathematical modeling, Euler-Euler method, Euler-Lagrange method, physical and chemical processes.

The research was financially supported by the RFBR (project no. 18-38-00775_мол_a).

\section{REFERNCES}

1. Liu Q., Zhong W., Shao Y., Yu A. Research trends and recent advances of oxyfuel combustion of solid fuels in fluidized beds. CIESC Journal, 2019, vol. 3, pp. 1-23.

2. Energeticheskaya strategiya Rossii na period do 2030 goda [Energy Strategy of Russia for the period up to 2030 (ES-2030)] approved by decree №1715-r of the Government of the Russian Federation dated 13 November 2009]. Ministry of Energy of the RF. Available at: https://minenergo.gov.ru/node/1026 (accessed 12 November 2019).

3. Myöhänen K. Modelling of combustion and sorbent reactions in three-dimensional flow environment of a circulating fluidized bed furnace. Dr.Sc. Diss. Lappeenranta, 2011. 164 p.

4. Agrawal K., Loezos P.N., Syamlal M., Sundaresan S. The role of mesoscale structures in rapid gas-solid flow. Journal of Fluid Mechanics, 2001, vol. 445, pp. 151-185.

5. Reh L. Development potentials and research needs in circulating fluidized bed combustion. China Particuology, 2003, vol. 1, no. 5, pp. $185-200$

6. Ibsen C.H., Helland E., Hjertager B.H., Solberg T., Tadrist L., Occelli R. Comparison of multifluid and discrete particle modelling in numerical predictions of gas particle flow in circulating fluidised beds. Powder Technology, 2004, vol. 149, no. 1, pp. 29-41.

7. Andrews A.T., Loezos P.N., Sundaresan S. Coarse-Grid Simulation of Gas-Particle Flows in Vertical Risers. Industrial \& Engineering Chemistry Research, 2005, vol. 44, pp. 6022-6037.

8. Kallio S. Comparison of simulated and measured voidage an velocity profiles and fluctuations in a CFB riser. Proc. of the $8^{\text {th }}$
International Conference on Circulating Fluidized Beds. Hangzhou, China, 2005. pp. 105-112.

9. Igci Y., Andrews A.T., Sundaresan S., Pannala S., O'Brien T. Filtered two-fluid models for fluidized gas-particle suspensions. AIChe Journal, 2008, vol. 54, no. 6, pp. 1431-1448. Available at: https://doi.org/10.1002/aic.11481 (accessed 10 February 2020).

10. Wang S., Luo K., Hu C., Fan J. CFD-DEM study of the effect of cyclone arrangements on the gas-solid flow dynamics in the fullloop circulating fluidized bed. Chemical Engineering Science, 2017, vol. 172, pp. 199-215.

11. Tu Q., Wang H. CPFD study of a full-loop three-dimensional pilot-scale circulating fluidized bed based on EMMS drag model. Powder Technology, 2018, vol. 323, pp. 534-547.

12. Crowe C., Sommerfeld M., Tsuji Y. Multiphase flows with droplets and particles. Boca Raton, CRC Press, 1998. 496 p.

13. Deen N.G., Van Sint Annaland M., Van der Hoef M.A., Kuipers J.A.M. Review of discrete particle modeling of fluidized beds. Chemical Engineering Science, 2008, vol. 62, pp. 28-44.

14. Massoudi Farid M., Jeong H.J., Kim K.H., Lee J., Kim D., Hwang J. Numerical investigation of particle transport hydrodynamics and coal combustion in an industrial-scale circulating fluidized bed combustor: effects of coal feeder positions and coal feeding rates. Fuel, 2017, vol. 192, pp. 187-200.

15. Wu Y., Liu D., Zheng D., Ma J., Duan L., Chen X. Numerical simulation of circulating fluidized bed oxy-fuel combustion with Dense Discrete Phase Model. Fuel Processing Technology, 2019, vol. 195, pp. 1-14.

16. Goldschmidt M.J.V. Hydrodynamic modelling of fluidised bed spray granulation. Doctoral dissertation. Netherlands, 2001. 303 p. 
17. Hou B., Wang X., Zhang T., Li H. A model for improving the EulerEuler twophase flow theory to predict chemical reactions in circulating fluidized beds. Powder Technology, 2017, vol. 321, pp. 13-30.

18. Cardoso J., Silva V., Eusébio D., Brito P., Tarelho L. Improved numerical approaches to predict hydrodynamics in a pilot-scale bubbling fluidized bed biomass reactor: a numerical study with experimental validation. Energy Conversion and Management, 2018, vol. 156, pp. 53-67

19. Kallio S., Kilpinen P., Hupa M. Advanced modeling of nitrogen oxide emissions in circulating fluidized bed combustors: parametric study of hydrodynamics. $6^{\text {th }}$ Int. Conference on Circulating Fluidized Beds. Würzburg, 1999. pp. 659-664.

20. Kilpinen P., Kallio S., Hupa M., Advanced modeling of nitrogen oxide emissions in CFBC: parametric study of coal combustion and nitrogen compound chemistries. Proc. of the $15^{\text {th }}$ International Conference on FBC. Savannah, GA, USA, 1999. Paper No. FBC99-0155

21. Chapman S., Cowling T.G., Burnett D. The mathematical theor of non-uniform gases: an account of the kinetic theory of viscosity, thermal conduction and diffusion in gases. Cambridge, Cambridge university press, $1990.423 \mathrm{p}$

22. Benyahia S., Arastoopour H., Knowlton T.M., Massah H. Simulation of particles and gas flow behavior in the riser section of a circulating fluidized bed using the kinetic theory approach for the particulate phase. Powder Technology, 2000, vol. 112, no. 1-2, pp. 24-33.

23. Bird R.B., Stewart, W.E., Lightfoot E.N. Transport phenomena $\left(2^{\text {nd }}\right.$ ed.). New York, Wiley, 2002. $885 \mathrm{p}$

24. Taghipour F., Ellis N., Wong C. Experimental and computational study of gas-solid fluidized bed hydrodynamics. Chemical Engineering Science, 2005, vol. 60, pp. 6857-6867

25. Ergun S. Fluid flow through packed columns. Chemical Engineering Progress, 1952, vol. 48, pp. 89-94.

26. Wen C.Y., Yu Y.H. Mechanics of fluidization. Chemical Engineering Progress Symposium Series, 1966, vol. 62, pp. 100-111.

27. Qi H., You C., Boemer A., Renz U. Eulerian simulation of gassolid two-phase flow in a CFB-riser under consideration of cluster effects. Fluidization 2000: Science and Technology. Eds. D. Xu, S. Mori. Xi'an, Xi'an Publishing House, 2000. pp. 231-237.

28. Beetstra R., Van der Hoef M.A., Kuipers J.A.M. Numerical study of segregation using a new drag force correlation for polydisperse systems derived from lattice-Boltzmann simulations. Chemical Engineering Science, 2007, vol. 62, no. 1-2, pp. 246-255.

29. Yang N., Wang W., Ge W., Li J. CFD simulation of concurrent upgas solidflow in circulating fluidized beds with structuredependent drag coefficient. Chemical Engineering Journal, 2003, vol. 96, pp. 71-80.

30. Qi H., Li F., Xi B., You C. Modeling of drag with the Eulerian approachand EMMS theory for heterogeneous dense gas-solid two-phase flow. Chemical Engineering Science, 2007, vol. 62, pp. 1670-1681.

31. Li J., Kwauk M. Particle-fluid two-phase flow: the energyminimization multi-scale method. Beijing, Metallurgical Industry Press, 1994. $215 \mathrm{p}$.

32. Guangbo Z. A coal combustion model for circulating fluidized bed boilers. Fuel, 2000, vol. 79, pp. 165-172.

33. Gungor A. Hydrodynamic modeling of a circulating fluidized bed. Powder Technology, 2007, vol. 172, pp. 1-13.

34. Nakamura H. Numerical modeling of particle fluidization behavior in a rotating fluidized bed. Powder Technology, 2007, vol. 171, pp. 106-117.

35. Hadavand A. An innovative bed temperature-oriented modeling and robust control of a circulating fluidized bed combustor. Che mical Engineering Journal, 2008, vol. 140, pp. 497-508.

36. Kong L.X., Hodgson P.D. Computational simulation of gas flow and heat transfer near an immersed object in fluidized beds. $A d$ vances in Engineering Software, 2007, vol. 38, pp. 826-834.

37. Bastos J.C.S.C. Modelling and simulation of a gas-solids dispersion flow in a high flux circulating fluidized bed (HFCFB) riser. Catalysis Today, 2008, vol. 130, pp. 462-470
38. Cornelissen J.T. CFD modelling of a liquid-solid fluidized bed. Chemical Engineering Science, 2007, vol. 62, pp. 6334-6348

39. Adamczyk W.P., Wecel G., Klajny M., Kozołub P., Klimanek A., Białecki R.A. Modeling of particle transport and combustion phenomena in a large-scale circulating fluidized bed boiler using a hybrid Euler-Lagrange approach. Particuology, 2014, vol. 16, pp. $29-40$.

40. Bordbar M.H., Myohanen K., Hyppanen T. Coupling of a radiative heat transfer model and a three-dimensional combustion model for a circulating fluidized bed furnace. Applied Thermal Engineering, 2015, vol. 76, pp. 344-356.

41. Bordbar M.H., Wecel G., Hyppanen T. A line by line based weighted sum of gray gases model for inhomogeneous $\mathrm{CO}_{2}-\mathrm{H}_{2} \mathrm{O}$ mixture in oxy-fired combustion. Combustion and Flame, 2014, vol. 161, pp. 2435-2445.

42. Nies M., Plackmeyer J. Computer-aided optimisation of gasparticle flow and combustion at the duisburg circulating fluidised bed furnace. VGB PowerTech Journal, 2011, vol. 8, pp. 64-69.

43. Zhang N., Wang W., Li J. CFD simulation of combustion in a 150 MWe circulating fluidized bed boiler. Cleaner Combustion and Sustainable World - Proc. of the $7^{\text {th }}$ International Symposium on Coal Combustion. Eds. H. Qi, B. Zhao. Berlin, Springer-Verlag, 2013. pp. 65-67.

44. Myöhänen K., Hyppänen T. A three-dimensional model frame for modelling combustion and gasification in circulating fluidized bed furnaces. International Journal of Chemical Reactor Engineering, 2011, vol. 9, no. 1, pp. 1-55.

45. Wischnewski R., Ratschow L., Hartge E.U., Werther J. Reactive gas-solids flows in large volumes-3D modeling of industrial circulating fluidized bed combustors. Particulogy, 2010, vol. 8, pp. 67-77.

46. Pallares D., Palonen M., Yla-Outinen V., Johnsson F. Modeling of the heat transfer in large-scale circulating fluidized bed furnaces. Proc. of the $21^{\text {st }}$ International Conference on Fluidized Bed Combustion. Eds. U. Arena, R. Chirone, M. Miccio, P. Salatino. EnzoAlbanoEditore, Naples, 2012. pp. 867-874.

47. ANSYS FLUENT 12.0, Theory Guide. 2009. Available at: https:// www.afs.enea.it/project/neptunius/docs/fluent/html/th/main_pre.ht $\mathrm{m}$ (accessed 12 November 2019).

48. Salomatov V.V., Gil A.V., Starchenko A.V., Arkhipov R.Yu. Chislennoe issledovanie vliyaniya dispersnogo sostava na harakteristiki szhiganiya ugol'nogo topliva $\mathrm{v}$ cirkuliruyushchem kipyashchem sloe [Numerical study of the influence of disperse composition on the characteristics of coal fuel combustion in a circulating fluidized bed]. Vestnik Novosibirskogo gosudarstvennogo universiteta. Seriya: Physika, 2016, vol. 11, no. 3, pp. 53-61.

49. Gu J., Shao Y., Liu X., Zhong W., Yu A. Modelling of particle flow in a dual circulation fluidized bed by a Eulerian-Lagrangian approach. Chemical Engineering Science, 2018, vol. 192, pp. 619-633.

50. Amarasinghe S., Jayarathna K., Ahangama S., Moldestad M.E., Tokheim L.-A. Experimental study and CFD modelling of minimum fluidization velocity for Geldart A, B and D particles. International Journal of Modeling and Optimization, 2017, vol. 7, pp. 152-156.

51. Bandara J.C., Eikeland M.S., Moldestad B.M.E. Analyzing the effects of particle density, size, size distribution and shape for minimum fluidization velocity with Eulerian-Lagrangian, CFD simulation. Proc. of the $58^{\text {th }}$ Conference on Simulation and Modelling. Reykjavik, Iceland, 2017. pp. 60-65.

52. Koksharev O.M. Analiz primenimosti chislennykh algoritmov dlya modelirovaniya protsessov $v$ topke kotla s tsirkuliruyushchim kipyashchim sloem. Diss. magistra [Analysis of the applicability of numerical algorithms for modeling processes in the furnace of a boiler with a circulating fluidized bed. Master's thesis]. Tomsk, 2017. $125 \mathrm{p}$.

53. Suik H., Pihu T. Warranty reliability of CFB boiler burning oil shale. Oil Shale, 2009, vol. 26, no. 2, pp. 99-107.

Received: 22 February 2020.

Information about the authors

Andrey V. Gil, Cand. Sc., associate professor, National Research Tomsk Polytechnic University.

Alexander S. Zavorin, Dr. Sc., professor, National Research Tomsk Polytechnic University.

Oleg M. Koksharev, postgraduate, National Research Tomsk Polytechnic University. 